\title{
Correlation between RASSF1A, P16, DAP Kinase Promoter Hypermethylation and Lung Cancer: Relation with Smoking Status
}

Elmabruk Abdullah Gamag*

Faculty of Medical Technology, Sabratha University, Libya

DOI: $10.36348 /$ sjmps.2020.v06i02.013 $\quad$ | Received: 06.02.2020 | Accepted: 14.02 .2020 | Published: 27.02 .2020

*Corresponding author: Elmabruk Abdullah Gamag

\section{Abstract}

Epigenetic silencing of tumor suppressor genes (TSG) is a fundamental incident in the pathogenesis of human cancer. Inactivation of TSG is accomplished by aberrant chromatin modifications including DNA hypermethylation of the gene promoter. Of the most frequently hypermethylated TSG, Ras Association Domain Family1 (RASSF1A), P16 and Death Association Protein kinase $(D A P K)$ genes. Aberrant hypermethylation of these genes have been correlated with non small cell lung cancer (NSCLC) promoting disease recurrence and remote metastasis. This review aims to provide the readers with a precise description of the research to date in the field of epigenetics and its impact on people with NSCLCs. The focus of this study will be on promoter hypermethylation of three different lung cancer associated genes P16, RASSF1A and $D A P K$ and how these genes inactivated and contribute to the pathogenecity of human malignancies. Moreover, The study aims to investigate the impact of tobacco smoke on the hypermethylation frequency of the mentioned genes. Thus, the promoter hypermethylation frequency could be a promising biomarker to improve NSCLC diagnosis and screening. Keywords: Non small cell lung cancer, DNA methylation; Tumor suppressor genes; RASSF1A; P16, DAPK.

Copyright @ 2020: This is an open-access article distributed under the terms of the Creative Commons Attribution license which permits unrestricted use, distribution, and reproduction in any medium for non-commercial use (NonCommercial, or CC-BY-NC) provided the original author and source are credited.

\section{INTRODUCTION}

Lung cancer is responsible for cancer related deaths and one of most common malignancies worldwide [1]. Millions of people died due to lung cancer each year, and more than a million of people are annually diagnosed with lung cancer [2]. In addition, the survival rate is about $16 \%$ for most of the cases diagnosed with lung cancer [3]. These percentages highlighted the disease as a major public health panic. On the other hand, an early detection of the disease predisposes for a significant increase in survival rate [4]. Approximately half of the patients with non-small cell lung cancer (NSCLC) were not diagnosed at an early stages, where treatment is curative [3]. Lung cancer is divided into two major groups non small cell lung cancer (NSCLC) and small cell lung cancer, NSCLC is account for approximately $85 \%$ of lung cancer, and sub divided into adenocarcinoma, squamous cell carcinoma and large cell carcinoma [5].

\section{Effects of Environmental Carcinogenesis on Epigenetics}

Epigenetic patterns found to be altered by several epimutagens in the environment and diet [6]. Numerous environmental factors including chemicals found in cigarette smoke and alcohol consumption as well as dietary contaminants play a crucial role in the etiology of human cancer [7, 8]. Other life style factors such as plutonium or radon exposure and helicobacter pylori infections are thought to be involved in the development of broad spectrum of human cancers including NSCLC [9]. More importantly, tobacco smoking is considered to be the most life style that contribute to cancer development, and reported to be the most common cause of lung cancer worldwide [10]. It is argued that the overwhelming majority of patients with lung cancer are indicated to have smoking status [11], estimating that as many as 69 of 4800 identified compounds of a cigarette may possibly be carcinogens [12].

\section{Challenges Faced the Efficiency of Lung Cancer Treatment}

It is widely accepted that lung cancer faced noticeable challenges to diagnose early and treat efficiently regardless of the reality that most of the causes of lung cancer are well recognized, in addition to the remarkable advance in the molecular mechanisms underlying lung carcinogenesis [9]. Lung cancer is a deadly disease and the five year survival rates at approximately $17 \%$, despite the advanced diagnostic strategies and development of innovative treatment 
[13]. More importantly, treatment failure and death for NSCLC patients are mainly due to disease recurrence and remote metastasis [14]. It is therefore of critical urgency to detect lung cancer at an early stage with the current innovative technology using suitable molecular markers as an alternative approach. These Specific biomarkers could play a significant role in determining pathological changes at an early stage and facilitate clinical intervention of the lung cancer [4].

\section{Role of Epigenetics in Lung Cancer}

It is commonly known that lung cancer is a genetic disease, however; etiology of lung cancer is not only affected by genetic factors such as mutations and genetic polymorphisms but also the effect of epigenetic changes has been implicated in lung cancer [9]. Determining where and when a specific gene to expressed during development is influenced by epigenetic modifications, which are heritable during cell division without alterations in DNA sequence [15]. Therefore, the initiation and progression of cancer triggered by acquired epigenetic alterations through modulating gene expression [16]. Methylation of genomic promoter region is the most studied epigenetic mechanisms whereby cytosines at position 5 in $\mathrm{CpG}$ dinucleotides are methylated [17]. Promoter methylation of tumor suppressor genes (TSG) predisposes for frequent alterations on several types of human cancers including NSCLC [9].

Epigenetic silencing of tumor suppressor genes is a fundamental incident in the pathogenesis of human cancer [18]. One of the main mechanisms to inactivate TSG is CpG promoter hypermethylation of a gene [19]. Such alterations are sensitive and specific biomarkers to a wide variety of tumor cells [20]. Furthermore, aberrant DNA methylation of normally unmethylated CpG-islands within or near the promoter region is associated with the transcription silencing of tumor suppressor genes in human cancers including lung cancer. Additionally, inactivation of TSG is accomplished by aberrant chromatin modifications including hypermethylation of the promoter region of a gene [18]. Several studies have illustrated the diagnostic effectiveness of DNA hypermethylation of a broad spectrum of well known tumor related genes such as p16, RASSF1, APC, MGMT, DAPK, GATA5, and $H O X 9$, in various biofluids, including bronchial aspirates, sputum, serum, plasma, and cell free circulating DNA [21]. Hypermethylation of three different lung cancer associated genes P16, RASSF1A and $D A P K$ will be discussed in the following paragraphs.

\section{Hypermethylation of Ras Association Domain Family1 (RASSF1A) Gene in Lung Cancer}

RASSF $1 A$ is a tumor suppressor gene that are epigenetically inactivated and contribute to pathogenesis and prognosis of human tumours [22].
Hypermethylation of CPG promoter region is a common cause of RASSF1A gene expression inactivation [23]. Inactivation of the gene expression is significantly affected by promoter hypermethylaion rather than mutation of RASSF1A gene [24]. Moreover, methylation of RASSFIA gene is rarely expressed in normal tissue, whereas the frequency of CPG methylation in the promoter region rises dramatically in tumor tissues [24]. It is found that the frequency of DNA hypermethylation of $\mathrm{CpG}$ islands ranges from 99\% in tumor tissue compared $0 \%$ in normal tissues, with the highest frequency of approximately $88 \%$ in lung cancer [25]. Hence, RASSF1A gene is one of the most frequently hypermethylated tumor suppressor genes in NSCLC and could be a significant biomarker for cancer detection.

\section{Hypermethylation of P16 Gene in Lung Cancer}

$P 16$ is an essential tumor suppressor gene that play a potential role in cell cycle regulation, and the CPG promoter hypermethylation of P16 is a frequent occurrence in human tumors [26]. The higher frequency of $P 16$ hypermethylation was reported in various types of human cancers including NSCLC, in which approximately $40 \%$ of the lung cancers exhibited p16 methylation [27]. In addition, about 22-60\% loss expression of p16 is correlated with several mechanisms together with promoter hypermethylation [28]. In 22 cases with $p 16$ gene hypermethylation that found in tumor tissues, only $4(18.1 \%)$ cases exhibited hypermethylation status in normal tissues, suggesting that tumor cells show more p16 hypermethylation frequency than that in neighboring normal cells [29]. It is therefore of important value to use gene promoter methylation of P16 detected in serum or sputum as a biomarker for NSCLC diagnosis [30].

\section{Hypermethylation of Death Association Protein Kinase $(D A P K)$ in Lung Cancer}

A significant correlation between the death association protein kinase $(D A P K)$ hypermethylation and NSCLC has been reported in several studies. Tang et al., illustrated that $44 \%$ of the examined tumors were hypermethylated at the CPG region of DAP- kinase [31]. Additionally, Yang et al., reported that the frequency of $D A P K$ methylation was considerably higher in NSCLC than peficancerous tissue [32]. Furthermore, in 2148 patients with NSCLC pooled from 28 studies, 870 cases were reported with $D A P$ kinase CPG promoter hypermethylation, the rate of hypermethylation was $40.50 \%$, and about $5.69 \%$ times higher than the one in normal lung tissues. Consequently, the risk of NSCLC was significantly correlated with $D A P K$ promoter hypermethylation [33]. Promoter hypermethylation of $D A P K$ in an early stage of tumor cells confirms the importance of this epigenetic abnormality in NSCLC [31]. 
Silencing of the tumor suppressor gene $D A P K$ through methylation plays an essential role in lung cancer pathogenecity, which could be due to the higher frequency rate of methylation found in malignant lesions [5]. These findings indicated the previously reported data by Yanagawa et al., in which methylation of $D A P K$ gene exhibited a higher frequency rate in NSCLCs than in non neoplastic lung tissue [2]. The 5 year survival rate of lung cancer patients with $D A P$ kinase methylation is lower than that in patients with unmethylated DAP kinase gene [21]. Inactivation of DAPK may be a remarkable biomarker for the molecular classification of stage I NSCLC [31].

\section{Impact of Tobacco Smoke on Methylation Levels of RASSF1A, P16 and Dap kinase Genes}

Several recent studies demonstrated the correlation between promoter hypermethylation of a number of genes and smoking behavior [6]. RASSF1A is one of the genes that frequently altered by CPG promoter methylation in smokers with lung cancer [34]. These results were consistent with another study which showed a higher methylation density of RASSF 1A gene of former smokers compared to both current smokers and non smokers, indicating significant responsive of RASSF1A methylation to smoking status [24]. Also, Kim et al., found that hypermethylation of RASSFIA promoter occurred more frequently in patients who started smoking early in their life. Moreover; hypermethylation could affect the survival of patients with NSCLC [10].

P16 gene also found to be a fundamental target to genetic changes in lung cancer pathogenesis of smokers [35]. Hypermethylation of $\mathrm{CpG}$ promoter region of $P 16$ appeared to be influenced by smoking status with a significant higher frequency compared to non smokers [36]. In addition, Jarmalaite et al., reported an interesting finding in which the frequency of p16 methylation of former smokers was significantly higher than that of current smokers [27]. Moreover; a remarkable association was shown between smoking characteristics such as duration of smoking or time since quitting and P16 methylation, which may contribute to the increased incidence of NSCLC [37]. However, Jarmalaite et al. demonstrated that no significant correlation was found between cumulative exposure to tobacco or duration of smoking and methylation [27]. Another noticeable finding exhibited that the occurrence of P16 hypermethylation was similar for individuals who started smoking before 19 years of age and individuals who begun smoking after 19 years10. On the other hand, the hypermethylation prevalence of RASSF1A gene was shown to be more frequent in those who started smoking while young [10]. The carcinogenic effect of tobacco smoke exerts by silencing tumor suppressor pathways through genetic and epigenetic mechanism [6].
Accumulating data reported no correlation between $D A P$ kinase promoter methylation and smoking behavior. Soria et al., showed no correlation between $\mathrm{CpG}$ promoter methylation and the smoking behavior [19]. Consistently, Yanagawa et al., argued that the frequency of alterations of DAP Kinase methylation did not change with the smoking status compared to RASSF1A and P16 [34]. Moreover, no association was noted between $D A P K$ promoter hypermethylation and tobacco smoke despite the reality that hypermethylation is an early incident of carcinogenesis and the significant role of smoking on development of NSCLC [33]. Further studies to gain a precise insight about the correlation between promoter methylation of these genes and smoking status are required.

It is believed that hypermethylation of a gene in individuals who started smoking early in life occurred in a gene specific manner, also; the nonrandom changes in the environment around a gene could influence the susceptibility to hypermethylation [10]. The raised activity of DNA methyltransferase is a common factor for the increased susceptibility to the promoter hypermethylation of a specific gene in those who started smoking while young [38]. Cigarette smoke contains abundant carcinogens such as nitrosamine 4(methylnitro-samino)-1-(3-pyridyl)-1-butanone (also known as nicotine-derived nitrosamine ketone; NNK), these ingredients systemically stimulate lung tumors [39]. NNK triggers methyltransferase accumulation and the subsequent promoter hypermethylation of TSG will possibly lead to tumorigenesis and offers a significant association between tobacco smoking and lung cancer [40]. Clinical studies described a potential correlation between promoter hypermethylation and tobacco smoke at approximately 20 TSGs in lung tumors [41].

\section{Conclusion}

The prevalence of hypermethylation of $\mathrm{CPG}$ promoter region of the described genes was consistent. Correlation between promoter methylation of these genes and lung cancer has been indicated since the promoter hypermethylation was crucially different between cancer and normal control tissues. Hypermethylation of both P16 and RASSF1A genes was significantly correlated with tobacco smoke and drives a potential impact on patients with NSCLC. Understanding the accurate epigenetic signal at promoter region will certainly be of great clinical benefit. Hence, these findings recommend that methylation of P16, RASSFA1 and DAP kinase could be a promising biomarker to improve the early detection of NSCLC and subsequent management of individuals with diagnosed malignancy. Further research to establish the underlying mechanisms for promoter hypermethylation in tobacco induced cancer, and to overcome challenges facing development of epigenetic 
biomarkers that will improve prognostication and direct for the appropriate use of cancer therapies.

\section{REFERENCES}

1. Esteller, M., Sanchez-Cespedes, M., Rosell, R., Sidransky, D., Baylin, S. B., \& Herman, J. G. (1999). Detection of aberrant promoter hypermethylation of tumor suppressor genes in serum DNA from non-small cell lung cancer patients. Cancer research, 59(1), 67-70.

2. Yanagawa, N., Tamura, G., Oizumi, H., Takahashi, N., Shimazaki, Y., \& Motoyama, T. (2003). Promoter hypermethylation of tumor suppressor and tumor-related genes in non-small cell lung cancers. Cancer science, 94(7), 589-592.

3. Levy, M. A., Lovly, C. M., \& Pao, W. (2012). Translating genomic information into clinical medicine: lung cancer as a paradigm. Genome research, 22(11), 2101-2108.

4. Lianidou, E., \& Alix-Panabières, C. (2017). Early detection of lung cancer based on DNA methylation analysis in sputum and plasma. Transl Cancer Res, 6, S1.

5. Li, Y., Zhu, M., Zhang, X., Cheng, D., \& Ma, X. (2015). Clinical significance of DAPK promoter hypermethylation in lung cancer: a metaanalysis. Drug design, development and therapy, 9, 1785-1796.

6. Herceg, Z. (2007). Epigenetics and cancer: towards an evaluation of the impact of environmental and dietary factors. Mutagenesis, 22(2), 91-103.

7. Mathers, J. C. (2004). The biological revolutiontowards a mechanistic understanding of the impact of diet on cancer risk. Mutation Research/Fundamental and Molecular Mechanisms of Mutagenesis, 551(1-2), 43-49.

8. Peto, J. (2001). Cancer epidemiology in the last century and the next decade. Nature, 411(6835), 390-395.

9. Vaissière, T., Hung, R. J., Zaridze, D., Moukeria, A., Cuenin, C., Fasolo, V., ... \& Tost, J. (2009). Quantitative analysis of DNA methylation profiles in lung cancer identifies aberrant DNA methylation of specific genes and its association with gender and cancer risk factors. Cancer research, 69(1), 243-252.

10. Kim, D. H., Kim, J. S., Ji, Y. I., Shim, Y. M., Kim, H., Han, J., \& Park, J. (2003). Hypermethylation of RASSF1A promoter is associated with the age at starting smoking and a poor prognosis in primary non-small cell lung cancer. Cancer research, 63(13), 3743-3746.

11. Lyn-Cook, L., Word, B., George, N., Lyn-Cook, B., \& Hammons, G. (2014). Effect of cigarette smoke condensate on gene promoter methylation in human lung cells. Tobacco induced diseases, 12(1), 15.
12. Hoffmann, D., Hoffmann, I., \& El-Bayoumy, K. (2001). The Less Harmful Cigarette: A Controversial Issue. A Tribute to Ernst L. Wynder. Chemical research in toxicology. 14:767-90.

13. Cortés, R., Tarrado-Castellarnau, M., Talancón, D., López, C., Link, W., Ruiz, D., ... \& Cascante, M. (2014). A novel cyclometallated Pt (II)ferrocene complex induces nuclear FOXO3a localization and apoptosis and synergizes with cisplatin to inhibit lung cancer cell proliferation. Metallomics, 6(3), 622-633.

14. Mainini, C., Rebelo, P. F., Bardelli, R., Kopliku, B., Tenconi, S., Costi, S., ... \& Fugazzaro, S. (2016). Perioperative physical exercise interventions for patients undergoing lung cancer surgery: What is the evidence?.SAGE open medicine, 4, 2050312116673855.

15. Bowman, R. V., Yang, I. A., Semmler, A. B., \& Fong, K. M. (2006). Epigenetics of lung cancer. Respirology, 11(4), 355-365.

16. Palmisano, W. A., Divine, K. K., Saccomanno, G., Gilliland, F. D., Baylin, S. B., Herman, J. G., \& Belinsky, S. A. (2000). Predicting lung cancer by detecting aberrant promoter methylation in sputum. Cancer research, 60(21), 5954-5958.

17. Adorján, P., Distler, J., Lipscher, E., Model, F., Müller, J., Pelet, C., ... \& Howe, A. (2002). Tumour class prediction and discovery by microarray-based DNA methylation analysis. Nucleic acids research, 30(5), e21.

18. Dammann, R. H., Richter, A. M., Jiménez, A. P., Woods, M., Küster, M., \& Witharana, C. (2017). Impact of natural compounds on DNA methylation levels of the tumor suppressor gene RASSF1A in cancer. International journal of molecular sciences, 18(10), 2160.

19. Soria, J. C., Rodriguez, M., Liu, D. D., Lee, J. J., Hong, W. K., \& Mao, L. (2002). Aberrant promoter methylation of multiple genes in bronchial brush samples from former cigarette smokers. Cancer Research, 62(2), 351-355.

20. Kajiura, K., Masuda, K., Naruto, T., Kohmoto, T., Watabnabe, M., Tsuboi, M., ... \& Imoto, I. (2017). Frequent silencing of the candidate tumor suppressor TRIM58 by promoter methylation in early-stage adenocarcinoma. Oncotarget, 8(2), 2890

21. Zhang, Y., Elgizouli, M., Schöttker, B., Holleczek, B., Nieters, A., \& Brenner, H. (2016). Smokingassociated DNA methylation markers predict lung cancer incidence. Clinical epigenetics, 8(1), 127.

22. van der Weyden, L., \& Adams, D. J. (2007). The Ras-association domain family (RASSF) members and their role in human tumourigenesis. Biochimica et Biophysica Acta (BBA)-Reviews on Cancer, 1776(1), 58-85.

23. Zhang, Z., Yan, S., Cui, H., Chen, H., \& Liu, J. (2019). Correlation Between RASSF1A Gene Promoter Hypermethylation in Serum or Sputum 
and Non-Small Cell Lung Cancer (NSCLC): A Meta-Analysis. Medical science monitor: international medical journal of experimental and clinical research, 25, 5518-5524.

24. Han, J. C., Xu, F., Chen, N., Qi, G. B., Wei, Y. J., Li, H. B., .. \& Li, X. F. (2016). Promoter methylations of RASSF1A and p16 is associated with clinicopathological features in lung cancers. Journal of cancer research and therapeutics, 12(1), 340-349.

25. Donninger, H., Vos, M. D., \& Clark, G. J. (2007). The RASSF1A tumor suppressor. Journal of cell science, 120(18), 3163-3172.

26. Esteller, M., Corn, P. G., Baylin, S. B., \& Herman, J. G. (2001). A gene hypermethylation profile of human cancer. Cancer research,61(8), 32253229.

27. Jarmalaite, S., Kannio, A., Anttila, S., Lazutka, J. R., \& Husgafvel-Pursiainen, K. (2003). Aberrant p16 promoter methylation in smokers and former smokers with nonsmall cell lung cancer. International journal of cancer, 106(6), 913-918.

28. Gu, J., Wen, Y., Zhu, S., Hua, F., Zhao, H., Xu, H., ... \& Zhou, Q. (2013). Association between P16INK4a promoter methylation and non-small cell lung cancer: a meta-analysis. PloS one, 8(4):e60107.

29. Hong, Y. S., Roh, M. S., Kim, N. Y., Lee, H. J., Kim, H. K., Lee, K. E., ... \& Kim, J. Y. (2007). Hypermethylation of p16INK4a in Korean nonsmall cell lung cancer patients. Journal of Korean medical science, 22(Suppl), S32-S37.

30. Tuo, L., Sha, S., Huayu, Z., \& Du, K. (2018). P16 INK4a gene promoter methylation as a biomarker for the diagnosis of non-small cell lung cancer: An updated meta-analysis. Thoracic cancer, 9(8), 1032-1040.

31. Tang, X., Khuri, F. R., Lee, J. J., Kemp, B. L., Liu, D., Hong, W. K., \& Mao, L. (2000). Hypermethylation of the death-associated protein (DAP) kinase promoter and aggressiveness in stage I non-small-cell lung cancer. Journal of the National Cancer Institute, 92(18), 1511-1516.

32. Yang, X. Y., Zhang, J., Yu, X. L., Zheng, G. F., Zhao, F., \& Jia, X. J. (2018). Death-associated protein kinase promoter methylation correlates with clinicopathological and prognostic features in nonsmall cell lung cancer patients: A cohort study. Journal of cancer research and therapeutics, 14(8), 65.
33. Zhang, Y., Wu, J., Huang, G., \& Xu, S. (2018). Clinicopathological significance of DAPK promoter methylation in non-small-cell lung cancer: a systematic review and metaanalysis. Cancer management and research, 10, 6897-6904.

34. Yanagawa, N., Tamura, G., Oizumi, H., Endoh, M., Sadahiro, M., \& Motoyama, T. (2011). Inverse correlation between EGFR mutation and FHIT, RASSF1A and RUNX3 methylation in lung adenocarcinoma: relation with smoking status. Anticancer research, 31(4), 1211-1214.

35. Osada, H., \& Takahashi, T. (2002). Genetic alterations of multiple tumor suppressors and oncogenes in the carcinogenesis and progression of lung cancer. Oncogene, 21(48), 7421-7434.

36. Liu, Y., Lan, Q., Siegfried, J. M., Luketich, J. D., \& Keohavong, P. (2006). Aberrant promoter methylation of p16 and MGMT genes in lung tumors from smoking and never-smoking lung cancer patients. Neoplasia (New York, NY), 8(1), 46-51.

37. Kim, D. H., Nelson, H. H., Wiencke, J. K., Zheng, S., Christiani, D. C., Wain, J. C., ... \& Kelsey, K. T. (2001). p16INK4a and histology-specific methylation of $\mathrm{CpG}$ islands by exposure to tobacco smoke in non-small cell lung cancer. Cancer research, 61(8), 3419-3424.

38. Belinsky, S. A., Nikula, K. J., Baylin, S. B., \& Issa, J. P. (1996). Increased cytosine DNAmethyltransferase activity is target-cell-specific and an early event in lung cancer. Proceedings of the National Academy of Sciences, 93(9), 40454050.

39. Hecht, S. S. (2002). Cigarette smoking and lung cancer: chemical mechanisms and approaches to prevention. The lancet oncology, 3(8), 461-469.

40. Lin, R. K., Hsieh, Y. S., Lin, P., Hsu, H. S., Chen, C. Y., Tang, Y. A., ... \& Wang, Y. C. (2010). The tobacco-specific carcinogen NNK induces DNA methyltransferase 1 accumulation and tumor suppressor gene hypermethylation in mice and lung cancer patients. The Journal of clinical investigation, 120(2), 521-532.

41. Piperi, C., Vlastos, F., Farmaki, E., Martinet, N., \& Papavassiliou, A. G. (2008). Epigenetic effects of lung cancer predisposing factors impact on clinical diagnosis and prognosis. Journal of cellular and molecular medicine, 12(5a), 14951501. 